\title{
Antiperistaltic Segment for Profuse Ileostomy Diarrhoea
}

\author{
HAROLD ELLIS,* D.M., M.CH., F.R.C.S. ; IVAN COLL, † M.B., F.R.C.S.
}

Brit. med. F., 1968, 1, 556-557

Considerable interest has recently been shown in the use of a reversed segment of intestine to interrupt the normal sweep of peristalsis along the gut and thus to delay the bowel transit and emptying times. Technically the operation comprises division of a short segment of intestine, its rotation on its vascular pedicle through 180 degrees, and then its re-anastomosis either at the same place or at some other place along the alimentary canal.

So far the principal use of this procedure has been in the "short bowel syndrome" of diarrhoea, steatorrhoea, malabsorption, hypoproteinaemia, and weight loss which results from massive intestinal resection. We have reported preoperative and postoperative studies on a patient submitted to the reversed segment procedure who had previously undergone extensive bowel resection after a mesenteric embolism (Venables, Ellis, and Smith, 1966) and reviewed the seven previously published examples of this operation. Since then, Fink and Olson (1967) have published four further cases. Of these 12 patients (all of whom improved after reversal) five had had extensive resection for Crohn's disease, two for mesenteric vascular occlusion, three for adhesions or small-bowel volvulus, and one patient, recorded by Fink and Olson, had had at various times resections of his carcinoma of the stomach, caecum, and rectum, leaving him with only $9 \mathrm{ft}$. $(274 \mathrm{~cm}$.) of small intestine together with the transverse and upper part of the descending colon. In only one case so far reported was the length of the small intestine normal (Madding, Kennedy, and MacLaughlin, 1965); this patient was a woman of 67 with profuse diarrhoea of unknown origin.

In this paper we describe the successful use of an antiperistaltic segment of small intestine in the treatment of profuse lleostomy diarrhoea in a patient who had undergone total colectomy for polyposis coli. Both the length and the appearance of the small intestine in this case were normal.

\section{Case Report}

The patient was an unmarried woman of 23 . There was no family history of large-bowel polyps. Rectal bleeding and the passage of mucus in the stools were first noted at the age of 9 years. Medical advice was not obtained until she was 12 , by which time the discharge of blood and mucus had become considerable and polyps had begun to prolapse from the anal verge. A diagnosis of polyposis coli was made, the polyps being confined to the rectum and descending colon. At this stage a resection of the left colon and rectum was performed with transverse colostomy. Three years later polyps began to appear at the colostomy site and mucus and blood once again appeared in the stool. In 1960, when she was aged 16, the right side of the colon was resected and an ileostomy was performed.

For the next seven years her life was plagued by incessant diarrhoea through the ileostomy stoma, so that she required repeated admission to hospital for the treatment of excoriation of the skin of the whole abdominal wall and for episodes of dehydration. All the usual drugs, with dietary advice, were employed without avail, nor did a laparotomy and refashioning procedure on the ileostomy in 1963 have any effect on the incessant flow of ileostomy fluid.

During her repeated hospital admissions she became depressed and on three separate occasions attempted suicide.

On examination she was thin and depressed. There was an ileostomy stoma in the left iliac fossa surrounded by considerable excoriation of the abdominal wall. A continuous watery faecal discharge was observed emerging from the stoma. Blood count, serum electrolyte and protein estimations, and urine analysis were all normal.

We reoperated at the Addington Hospital, Durban, on 16 June 1967. The abdomen was explored by reopening the vertical lower abdominal excision. Apart from some adhesions there were no intra-abdominal abnormalities. The small intestine measured $18 \mathrm{ft} .(550 \mathrm{~cm}$.) and appeared normal, A 3-in. $(7.5-\mathrm{cm}$.) segment of ileum $12 \mathrm{in}$. ( $30 \mathrm{~cm}$.) above the ileostomy was mobilized on its vascular pedicle, divided at each end, rotated through 180 degrees, and anastomosed back in position to the ileum above and below with two layers of catgut. The bowel was wrapped in moist saline packs and left for 30 minutes. At the end of this time the initial slight cyanosis in the reversed segment had completely cleared, the bowel presented a normal colour, and the twisted vascular pedicle was pulsating normally. The abdomen was then closed in layers. Postoperative recovery was completely uneventful.

Six months after operation her condition showed a remarkable improvement. The ileostomy functioned three or four times daily, producing either a semi-solid or solid stool. The abdominal wall excoriation had completely healed. She led a normal social life, was back at work, and was considering matrimony.

Her weight increased from $96 \mathrm{lb}$. (43.5 kg.) preoperatively to $102 \mathrm{lb}$. (46.3 kg.).

Cineradiography of a barium meal and follow-through examination carried out two months after operation showed a rapid transi of the barium column from stomach to terminal ileum in only 15 minutes. On reaching the reversed segment the barium was seen to reflux so that the barium column was held up in the terminal ileum above the reversed segment.

\section{Discussion}

Though profuse diarrhoea immediately after the establishment of an ileostomy is almost invariable, subsequent weeks or months see a gradual thickening of the ileal content so that the discharge becomes semiformed and relatively manageable. This process is helped by simple measures such as the administration of kaolin, codeine, agar, and so on. For the diarrhoea to persist for years, bringing with it the accompaniments of skin excoriation, nutritional depletion, and complete destruction of the patient's morale, is fortunately rare, particularly in the presence, as in the above case, of a small intestine of normal length and structure. The dramatic improvement produced by an antiperistaltic segment stresses that this procedure might be applied to other problems of rapid intestinal transit time, apart from those resulting purely from massive resections of the small intestine. Indeed, we are reporting its successful use in a patient with severe post-vagotomy diarrhoea (Craft and Venables, in press).

It is well known from animal experiments that a long reversed segment of small intestine will produce a lethal functional obstruction of the bowel (Singleton and Rowe, 1954). The optimal length of the reversed segment in man remains to be determined, but from our own experience now of three cases, together with other published reports, an antiperistaltic segment of 3 to 4 in. $(7.5$ to $10 \mathrm{~cm}$.) appears to be effective. We would

- Department of Surgery, Westminster Hospital, London S.W.I.

† King Edward VIII Hospital, Durban, South Africa. 
advocate the precaution, used in the present case, of observing the reversed segment for some 30 minutes at operation after the anastomosis has been completed, in order to ensure that the blood supply of the reversed segment remains adequate after its vascular pedicle has been twisted through 180 degrees.

\section{Summary}

The use of a reversed segment of small intestine in the treatment of persistent profuse ileostomy diarrhoea after colectomy for polyposis coli is described. The value of the reversed intestinal segment procedure in patients with rapid intestinal transit time due to causes other than massive bowel resection warrants further exploration.

\section{REFERENCES}

Craft, I., and Venables, C. W. (1968). Ann. Surg. In press.
Fink, W. J., and Olson, J. D. (1967). Arch. Surg., 94, 700.

Madding, G. F., Kennedy, P. A., and MacLaughlin, R. T. (1965). Ann. Surg., 161, 601

Singleton, A. O., and Rowe, E. B. (1954). Ann. Surg., 139, 853.

Venables, C. W., Ellis, H., and Smith, A. D. M. (1966). Lancet, 2, 1390.

\title{
Synacthen Depot-Adrenal Response in Normal Subjects and Corticotrophin-treated Patients*
}

\author{
J. K. NELSON, M.D., M.R.C.P.ED. ; D. W. NEILL, M.SC., M.C.PATH., F.R.I.C. \\ D. A. D. MONTGOMERY, M.B.E., M.D., F.R.C.P. ; J. S. MACKAY, B.SC. \\ B. SHERIDAN, A.I.M.L.T. ; J. A. WEAVER, M.D., M.R.C.P.
}

Brit. med. 9., 1968, 1, 557-558

The synthetic polypeptide tetracosactrin, $B^{1-24}$ (Synacthen) consists of the $24 \mathrm{~N}$-terminal amino-acids of corticotrophin. Corticotrophin itself consists of a chain of 39 amino-acids, but it has been shown that biological activity resides in the first 19 amino-acids ( $\mathrm{Li}$ et al., 1960), whereas immunological activity and species specificity reside in the region of the 25th to 39th amino-acids ( $\mathrm{Li}, 1961$; Fleischer et al., 1965).

Hitherto, Synacthen has been used as a test of adrenocortical function in which the response of plasma 11-hydroxycorticosteroids (11-OHCS) is taken as an index of adrenal activity. The effect of a single intramuscular injection of aqueous Synacthen is short-lived, lasting about 90 minutes (ElShaboury, 1965). Recently a depot preparation (42,915-Ba, Ciba), Synacthen, complexed with zinc has been prepared, and we have investigated its use in normal subjects and also compared the response to Synacthen Depot and corticotrophin gel in rheumatoid and asthmatic patients.

\section{Subjects and Methods}

Normal controls for the dose response studies were six healthy medical students. A number of patients on long-term intermittent corticotrophin treatment (Nelson et al., 1966)
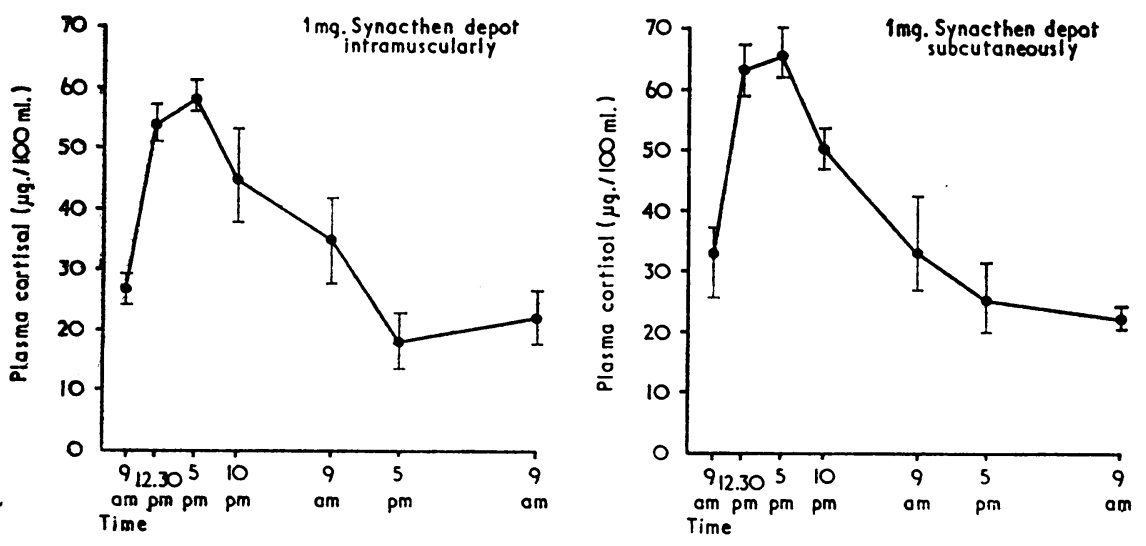

FIG. 1.-Mean plasma 11-OHCS levels ( $t$ standard error) in six normal subiects after administration of Synacthen Depot 1 mg. intramuscularly or subcutaneously. volunteered to try the new preparation, and 10 were studied as ward patients. Plasma 11-OHCS were estimated by a modification of the fluorometric procedure of Mattingly (1962). Venous blood samples for estimation of plasma 11-OHCS were drawn at 9 a.m. (patients) and 9 a.m. (students), after which times the Synacthen Depot was given; further blood samples were taken at 12.30 and 5 p.m. (for both patients and students), 10 p.m. (students), and 11.30 p.m. (patients). The tests were continued on the day after the Synacthen Depot or corticotrophin administration to study the effect of the previous day's treatment on the pituitary circadian rhythm.

\section{Results}

Normal Subjects.-A single injection of $1 \mathrm{mg}$. of Synacthen Depot had a similar effect whether given by intramuscular or subcutaneous injection (Fig. 1). There was a marked increment of 27.5 and $31.6 \mu \mathrm{g} . / 100 \mathrm{ml}$. respectively in the plasma 11OHCS levels at $3 \frac{1}{2}$ hours, and this was sustained to even slightly higher concentrations at eight hours. The levels at 10 p.m. (13 hours after injection) were still markedly elevated at the time of the expected daily decline in plasma cortisol. On the second day the pattern of plasma 11-OHCS showed the expected fall throughout the day between 9 a.m. and 5 p.m. The dose response curves (Fig. 2) showed that with $0.5 \mathrm{mg}$. of Synacthen Depot the pattern of response in the first 24 hours was the same as that given by greater doses, though the actual plasma 11-OHCS increments were naturally less. The largest dose of $2 \mathrm{mg}$. evoked an increase at $3 \frac{1}{2}$ hours and eight hours approximately similar to $1 \mathrm{mg}$. Thereafter the pattern of response was markedly different, showing a greater elevation for a longer time-the 13-, 24-, and 32-hour levels demonstrating sustained augmentation of adrenal response. Four of the subjects experienced slight dis-

\footnotetext{
From the Sir George B. Clark Metabolic Unit and Biochemistry Department, Royal Victoria Hos pital, Belfast.
} 